\title{
Prime filters of hyperlattices
}

\author{
R. Ameri, M. Amiri-Bideshki, A. B. Saeid and \\ S. Hoskova-Mayerova
}

\begin{abstract}
The purpose of this paper is the study of prime ideals and prime filters in hyperlattices. $I$-filter and the filter generated by $a \in L$ are introduced. Moreover, we introduce dual distributive hyperlattices, and $I$-filter in dual distributive hyperlattices. Some properties of hyperlattices are studied and the relationship between prime ideals and prime filters in hyperlattices is discussed.
\end{abstract}

\section{Introduction}

Hyperstructures theory was firstly introduced by F. Marty in the eighth congress of Scandinavians in 1934 [16]. This theory has been developed in various fields. The theory of hyperfields and hyperrings was initiated by Krasner and the results were published in 1983 in [15]. In the hyperring $(H,+, \cdot),(+)$ is a hyperoperation and (.) is a binary operation [15]. Schweigrt studied congruence of multialgebra [22]. Ameri and Nozari studied relationship between the categories of multialgebra and algebra [2]. Ameri and Rosenberg also studied congruences and strong congruences of multialgebras [3]. Hyperstructures were studied in many papers, e.g. $[1,7,8,9,10,12,18,17]$ and books e.g. $[7,24]$. Some applications can be found e.g. in [5, 6, 11, 23].

The theory of hyperlattices was introduced by Konstantinidou in 1977 [14]. Barghi considered the prime ideal theorem for distributive hyperlattices [19]. Koguep, Nkuimi, and Lele studied ideals and filters in hyperlattices [13]. Rasouli and Davvaz defined fundamental relation on a hyperlattice and obtained

Key Words: Hyperlattice, Prime ideal, Prime filter, Distributive element, Dual distributive element, Homomorphism.

2010 Mathematics Subject Classification: Primary 15A24, 15A60; Secondary 65F10.

Received: 07.05.2015

Accepted: 30.06 .2015 
a lattice from a hyperlattice. Moreover, they defined a topology on the set of prime ideals of a distributive hyperlattice [20,21].

In this paper, we introduce dual distributive hyperlattices, and $I$-filter in dual distributive hyperlattices and study their properties. We consider relationship between prime ideals and prime filters in hyperlattices. Moreover, $I$-filter and the filter generated by $a \in L$ are introduced.

\section{Preliminaries}

In this section we give some results of hyperstructures and mainly hyperlattices that we need to develop our paper.

Definition 2.1. [16] Let $H$ be a nonempty set and $P^{*}(H)$ denotes the set of all nonempty subsets of $H$. Maps $f: H \times H \longrightarrow P^{*}(H)$, are called hyperoperations.

Definition 2.2. [14] Let $L$ be a nonempty set, $\wedge-$ be a binary operation, and $\vee$ - be a hyperoperation on $L . L$ is called a hyperlattice if for all $a, b, c \in L$ the following conditions hold:

(i) $a \in a \vee a$, and $a \wedge a=a$;

(ii) $a \vee b=b \vee a$, and $a \wedge b=b \wedge a$;

(iii) $a \in[a \wedge(a \vee b)] \cap[a \vee(a \wedge b)]$;

(iv) $a \vee(b \vee c)=(a \vee b) \vee c$, and $a \wedge(b \wedge c)=(a \wedge b) \wedge c$;

(v) $a \in a \vee b \Longrightarrow a \wedge b=b$.

Let $A, B \subseteq L$. Then define:

$$
\begin{gathered}
A \vee B=\bigcup\{a \vee b \mid a \in A, b \in B\} ; \\
A \wedge B=\{a \wedge b \mid a \in A, b \in B\} .
\end{gathered}
$$

Definition 2.3. [13] Let $L$ be a hyperlattice. $L$ is called bounded if there exist $0,1 \in L$ such that for all $a \in L, 0 \leq a \leq 1$. We say that 0 is the least element and 1 is the greatest element of $L$.

Definition 2.4. [19] Let $L$ be a hyperlattice and $I$ be a nonempty subset of L. $I$ is called anideal if the following conditions hold:

(i) If $a, b \in I$, then $a \vee b \subseteq I$; 
(ii) If $a \in I, b \leq a$, and $b \in L$, then $b \in I$.

Ideal $I$ is called a prime ideal if $a \wedge b \in I$ then $a \in I$ or $b \in I$ for all $a, b \in L$.

Definition 2.5. [19] Let $L$ be a hyperlattice and $F$ be a nonempty subset of $L$. $F$ is called a filter if the following conditions hold:

(i) If $a, b \in F$, then $a \wedge b \in F$;

(ii) If $a \in F, a \leq b$, and $b \in L$, then $b \in F$.

Filter $F$ is called prime filter if $(a \vee b) \cap F \neq \emptyset$, then $a \in F$ or $b \in F$ for all $a, b \in L$.

Definition 2.6. [19] Let $L$ be a hyperlattice. $L$ is distributive if for all $a, b, c \in$ $L$ :

$$
a \wedge(b \vee c)=(a \wedge b) \vee(a \wedge c)
$$

Example 2.7. [13] Let $L=\{0, a, b, 1\}$ and $\wedge$ and $\vee$ are given with Table 1 . Then $(L, \vee, \wedge, 0,1)$ is a distributive hyperlattice.

\begin{tabular}{l|llll}
$\wedge$ & 0 & $a$ & $b$ & 1 \\
\hline 0 & 0 & 0 & 0 & 0 \\
$a$ & 0 & $a$ & 0 & $a$ \\
$b$ & 0 & 0 & $b$ & $b$ \\
1 & 0 & $a$ & $b$ & 1
\end{tabular}

(a)

\begin{tabular}{c|cccc}
$\vee$ & 0 & $a$ & $b$ & 1 \\
\hline 0 & $\{0\}$ & $\{a\}$ & $\{b\}$ & $\{1\}$ \\
$a$ & $\{a\}$ & $\{0, a\}$ & $\{1\}$ & $\{b, 1\}$ \\
$b$ & $\{b\}$ & $\{1\}$ & $\{0, b\}$ & $\{a, 1\}$ \\
1 & $\{1\}$ & $\{b, 1\}$ & $\{a, 1\}$ & $L$
\end{tabular}

(b)

Table 1

Theorem 2.8. [13] Let $L$ be a distributive hyperlattice. Then $0 \vee 0=0$.

Definition 2.9. [4] Let $L$ be a nonempty set. Then $L$ is called $\wedge-$ semilattice if for all $a, b, c \in L$ the following conditions hold:

(i) $a \wedge a=a$;

(ii) $a \wedge b=b \wedge a$;

(iii) $a \wedge(b \wedge c)=(a \wedge b) \wedge c$. 


\section{Ideals and filters in hyperlattices}

In this section, some properties of hyperlattices are studied and the relationship between prime ideals and prime filters in hyperlattices is discussed. Finally, $I$-filter and the filter generated by $a \in L$ are introduced.

In the sequel, $L$ denotes a bounded hyperlattice.

Remark 3.1. The converse of condition (v) in Definition 2.2 holds. By (iii) in Definition 2.2 and $a \wedge b=b$, we have $a \in a \vee(a \wedge b)=a \vee b$. Thus $a \in a \vee b$.

Definition 3.2. We define the order $\leq$ on $L$ by:

$$
a \leq b \Longleftrightarrow b \in a \vee b \Longleftrightarrow a \wedge b=a .
$$

Remark 3.3. The binary relation $\leq$ is reflexive, antisymmetric, and transitive. Thus $(L, \leq)$ is a poset.

Proposition 3.4. For all $a, b \in L$ the following conditions hold:

(i) $a \in a \vee 0$;

(ii) $1 \in a \vee 1$;

(iii) If $a, b \neq 0$ and $a \wedge b=0$, then $a, b \notin a \vee b$.

Proof. $(i),(i i): L$ is bounded, so $0 \leq a \leq 1$, for all $a \in L$. Therefore, by Definition 2.2(v), $a \in a \vee 0$, and $1 \in a \vee 1$.

(iii): Suppose $a \wedge b=0$. If $a \in a \vee b$, by Definition $2.2(\mathrm{v}), b=a \wedge b=0$. Thus $b=0$ which is a contradiction. If $b \in a \vee b$, we obtain that $a=0$, which is a contradiction.

Theorem 3.5. If $P$ is a prime ideal of $L$, then $L \backslash P$ is a prime filter of $L$.

Proof. Let $a, b \in L \backslash P$. We show that $a \wedge b \in L \backslash P$. It is clear that $a, b \notin P$. If $a \wedge b \in P$, then $a \in P$ or $b \in P$ because $P$ is prime filter, which is a contradiction. So $a \wedge b \in L \backslash P$. Assume $x \in L, a \in L \backslash P$ such that $a \leq x$, we show that $x \in L \backslash P$. It is clear that $a \notin P$. Suppose $x \notin L \backslash P$, so $x \in P$. We have $a \leq x$ and $P$ is an ideal, therefore $a \in P$ that is a contradiction. So, $\mathrm{L} \backslash P$ is a filter. It is enough to show that $L \backslash P$ is a prime filter. Suppose $a, b \in L$ and $(a \vee b) \cap(L \backslash P) \neq \emptyset$. So there exists $x \in L$ such that $x \in a \vee b$ and $x \in L \backslash P$. If $a, b \notin L \backslash P$, then $a, b \in P$, therefore $a \vee b \subseteq P$. Hence $(a \vee b) \cap(L \backslash P)=\emptyset$, which is a contradiction.

Corollary 3.6. If $P$ is a prime ideal of $L$, then $L \backslash P$ is a $\wedge$ - semilattice.

The converse of Theorem 3.5 holds. Now, we state it by the following theorem: 
Theorem 3.7. Let $F$ be a prime filter of $L$. Then $L \backslash F$ is a prime ideal of $L$.

Proof. Let $a, b \in L \backslash F$. If $a \vee b \nsubseteq L \backslash F$, then there exists $x \in a \vee b$ such that $x \notin L \backslash F$. So $x \in a \vee b$ and $x \in F$. Hence $x \in(a \vee b) \cap F$ and it implies that $(a \vee b) \cap F \neq \emptyset$. Thus $a \in F$ or $b \in F$, which is a contradiction. Let $a \in L \backslash F, x \leq a$. Assume $x \notin L \backslash F$. Thus we have: $x \in F$ and $x \leq a$. Since $F$ is a filter, $a \in F$, which is a contradiction. Therefore, $L \backslash F$ is an ideal. We must show that $L \backslash F$ is prime. Assume $a, b \in L$ such that $a \wedge b \in L \backslash F$. If $a \notin L \backslash F$ and $b \notin L \backslash F$, then $a \in F$ and $b \in F$. Since $F$ is a filter, $a \wedge b \in F$. So $a \wedge b \notin L \backslash F$, which is a contradiction. Thus $L \backslash F$ is a prime ideal.

In the following example, we show that $F$ must be prime in Theorem 3.7.

Example 3.8. Let $L=\{0, a, 1\}$ and $\wedge$ and $\vee$ are given with Table 2. Consider $F=\{1\}, F$ is a filter of $L$, but it is not prime. We have $L \backslash F=\{0, a\}, 0 \vee 0=L$, and $L \nsubseteq L \backslash F$, so $L \backslash F$ is not a ideal of $L$.

\begin{tabular}{l|lll}
$\wedge$ & 0 & $a$ & 1 \\
\hline 0 & 0 & 0 & 0 \\
$a$ & 0 & $a$ & $a$ \\
1 & 0 & $a$ & 1
\end{tabular}

(a)

\begin{tabular}{c|ccc}
$\vee$ & 0 & $a$ & 1 \\
\hline 0 & $\{0, a, 1\}$ & $\{a, 1\}$ & $\{1\}$ \\
$a$ & $\{a, 1\}$ & $\{a\}$ & $\{1\}$ \\
1 & $\{1\}$ & $\{1\}$ & $\{1\}$
\end{tabular}

(b)

Table 2

Theorem 3.9. Let $F$ be a non-empty subset of $L$ such that it is a $\wedge$-semilattice. Then $F$ is a prime filter if and only if for all $a, b \in L$, the following conditions hold:

(i) If $a \notin F$ and $b \notin F$, then $a \vee b \subseteq L \backslash F$;

(ii) If $a \in F$ and $x \in x \vee a$, then $x \in F$.

Proof. Suppose that $F$ is a prime filter. (i) If $a \notin F$ and $b \notin F$, then we have $a, b \in(L \backslash F)$. Since by Theorem 3.7, $(L \backslash F)$ is an ideal, $a \vee b \subseteq(L \backslash F)$.

(ii) Let $x \in x \vee a$. By Definition 3.2, $a \leq x$. Since $F$ is a filter, $a \leq x$, and $a \in F$, so $x \in F$.

Conversely, suppose $(a \vee b) \cap F \neq \emptyset$. If $a \notin F$ and $b \notin F$, by (i) we conclude that $a \vee b \subseteq(L \backslash F)$. Therefore $(a \vee b) \cap F=\emptyset$, which is a contradiction.

Definition 3.10. Let $L$ be a non-empty set and $\vee-$ be a hyperoperation on $L$. Then $L$ is called a $\vee-$ semi-hyperlattice if for all $a, b, c \in L$ the following conditions hold: 
(i) $a \in a \vee a$;

(ii) $a \vee b=b \vee a$;

(iii) $a \vee(b \vee c)=(a \vee b) \vee c$.

Example 3.11. Let $L=\{0, a, 1\}$. Hyperoperation $\vee-$ on $L$ is given by Table 3. Then $(L, \vee)$ is a $\vee$ - semi-hyperlattice.

\begin{tabular}{c|ccc}
$\vee$ & 0 & $a$ & 1 \\
\hline 0 & $\{0\}$ & $\{a, 1\}$ & $\{1\}$ \\
$a$ & $\{a, 1\}$ & $\{a\}$ & $\{1\}$ \\
1 & $\{1\}$ & $\{1\}$ & $\{1\}$
\end{tabular}

Table 3

Proposition 3.12. Let $L$ be $a \wedge-$ semilattice and $a \vee$ - semihyperlattice. Then $L$ is a hyperlattice if and only if for all $a, b \in L$ the following conditions hold:

(i) $a \in[a \vee(a \wedge b)] \cap[a \wedge(a \vee b)]$;

(i) $a \in a \vee b \Longrightarrow b \leq a$.

Definition 3.13. Let $(L, \vee, \wedge)$ be a hyperlattice and $S$ be a non-empty subset of $L$. Then $S$ is called a sub-hyperlattice of $L$ if $(S, \vee, \wedge)$ is a hyperlattice.

Example 3.14. Let $L$ be the hyperlattice in Example 3.8 and $S=\{a, 1\}$. Then $S$ is a sub-hyperlattice of $L$.

Proposition 3.15. Let $L$ be a hyperlattice and let $S$ be a non-empty subset of $L$. Then $S$ is a sub-hyperlattice of $L$ if and only if $a \wedge b \in S$ and $a \vee b \subseteq S$ for all $a, b \in S$.

Remark 3.16. Let $L$ be a distributive hyperlattice. $\{0\}$, and $L$ are subhyperlattice of $L$ which are called the trivial sub-hyperlattices of $L$. It is necessary that $L$ be a distributive hyperlattice. In Example 3.8, since $0 \vee 0=$ $\{0, a, 1\}$ and $0 \vee 0 \nsubseteq\{0\},\{0\}$ is not a sub-hyperlattice of $L$.

In this part we introduce $I$-filters and filters generated by an element $a$ in a type of hyperlattices that are called dual distributive.

Definition 3.17. A hyperlattice $L$ is called quasi dual distributive if for all $a, b, c \in L$.

$$
a \vee(b \wedge c) \subseteq(a \vee b) \wedge(a \vee c)
$$


$L$ is called weak dual distributive if:

$$
a \vee(b \wedge c) \cap[(a \vee b) \wedge(a \vee c)] \neq \emptyset ;
$$

$L$ is called dual distributive if:

$$
a \vee(b \wedge c)=(a \vee b) \wedge(a \vee c)
$$

Clearly a quasi dual distributive hyperlattice is weak dual distributive and a dual distributive hyperlattice is quasi distributive and weak dual distributive.

Example 3.18. (i) Let $L$ be the hyperlattice $L$ in Example 3.8. Then $(L, \vee, \wedge)$ is a dual distributive hyperlattice but it is not distributive. Since we have $0 \vee 0=L$, by Theorem $2.8, L$ isn't distributive.

(ii) Let $L=\{0, a, b, 1\} . \wedge$ operation and $\vee$ hyperoperation on $L$ are given by Table 4 . Then hyperlattice $L$ is not distributive, also $L$ is not dual distributive.

\begin{tabular}{l|llll}
$\wedge$ & 0 & $a$ & $b$ & 1 \\
\hline 0 & 0 & 0 & 0 & 0 \\
$a$ & 0 & $a$ & 0 & $a$ \\
$b$ & 0 & 0 & $b$ & $b$ \\
1 & 0 & $a$ & $b$ & 1
\end{tabular}

(a)

\begin{tabular}{c|cccc}
$\vee$ & 0 & $a$ & $b$ & 1 \\
\hline 0 & $\{0\}$ & $\{a, 1\}$ & $\{b, 1\}$ & $\{1\}$ \\
$a$ & $\{a, 1\}$ & $\{a, 1\}$ & $\{1\}$ & $\{1\}$ \\
$b$ & $\{b, 1\}$ & $\{1\}$ & $\{b, 1\}$ & $\{1\}$ \\
1 & $\{1\}$ & $\{1\}$ & $\{1\}$ & $\{1\}$
\end{tabular}

Table 4

Since $a \wedge(b \vee a)=a \wedge 1=a$ and $(a \wedge b) \vee(a \wedge a)=0 \vee a=\{a, 1\}$, therefore $L$ is not distributive and since $0 \vee(a \wedge b)=0 \vee 0=0$, and $(0 \vee a) \wedge(0 \vee b)=\{a, 1\} \wedge\{b, 1\}=\{0, a, b, 1\}, L$ is quasi dual distributive, but it is not dual distributive.

The hyperlattices such that are distributive and dual distributive, are called strongly distributive hyperlattices.

Example 3.19. Let $L=\{0, a, 1\}$. $\vee$ and $\wedge$ are given by Table 5 . Then $L$ is a strongly distributive hyperlattice.

Lemma 3.20. Let $L$ be a dual distributive hyperlattice and $a \in L$. We define $F_{a}=\{x \in L: x \neq 0,1 \in x \vee a\}$, then $F_{a}$ is a filter of $L$.

Proof. Suppose $x$ and $y \in F_{a}$. So $1 \in x \vee a$ and $1 \in y \vee a$. Hence $1 \in$ $(x \vee a) \wedge(y \vee a)$. Since $L$ is dual distributive, we have $a \vee(x \wedge y)=(a \vee x) \wedge(a \vee y)$. 


\begin{tabular}{l|lll}
$\wedge$ & 0 & $a$ & 1 \\
\hline 0 & 0 & 0 & 0 \\
$a$ & 0 & $a$ & $a$ \\
1 & 0 & $a$ & 1
\end{tabular}

(a)

\begin{tabular}{c|ccc}
$\vee$ & 0 & $a$ & 1 \\
\hline 0 & $\{0\}$ & $\{a, 1\}$ & $\{1\}$ \\
$a$ & $\{a, 1\}$ & $\{a\}$ & $\{1\}$ \\
1 & $\{1\}$ & $\{1\}$ & $\{1\}$
\end{tabular}

(b)

Table 5

So $1 \in(x \wedge y) \vee a$. Therefore, $(x \wedge y) \in F_{a}$. Let $x \in F_{a}$ and $y \in L$, such that $x \leq y$. Hence $1 \in x \vee a$ and $x \wedge y=x$. So we have $1 \in x \vee a=(x \wedge y) \vee a$. Since $L$ is dual distributive, $1 \in(a \vee x) \wedge(a \vee y)$. So $1 \in a \vee y$ and it implies that $y \in F_{a}$. Thus $F_{a}$ is a filter.

In Lemma $3.20, L$ must be dual distributive. In the following example we show that the converse of Lemma 3.20 does not hold.

Example 3.21. Let $L=\{0, a, b, 1\}$ and $\wedge$ be an operation and $\vee$ hyperoperation on $L$ are given with Table 6 .

\begin{tabular}{l|llll}
$\wedge$ & 0 & $a$ & $b$ & 1 \\
\hline 0 & 0 & 0 & 0 & 0 \\
$a$ & 0 & $a$ & $a$ & $a$ \\
$b$ & 0 & $a$ & $b$ & $b$ \\
1 & 0 & $a$ & $b$ & 1
\end{tabular}

(a)

\begin{tabular}{c|cccc}
$\vee$ & 0 & $a$ & $b$ & 1 \\
\hline 0 & $\{0, a, b, 1\}$ & $\{a, b, 1\}$ & $\{b\}$ & $\{1\}$ \\
$a$ & $\{a, b, 1\}$ & $\{a, b, 1\}$ & $\{b\}$ & $\{1\}$ \\
$b$ & $\{b\}$ & $\{b\}$ & $\{1, b\}$ & $\{1\}$ \\
1 & $\{1\}$ & $\{1\}$ & $\{1\}$ & $\{1\}$
\end{tabular}

(b)

Table 6: join table

Then $L$ is a hyperlattice which is not dual distributive, because we have $0 \mathrm{~V}$ $(a \wedge b)=0 \vee a=\{a, b, 1\}$, and $(0 \vee a) \wedge(0 \vee b)=\{a, b, 1\} \wedge b=\{a, b\}$. So, $0 \vee(a \wedge b) \neq(0 \vee a) \wedge(0 \vee b)$

We have $F_{a}=\{a, 1\}$. Since $a \in F_{a}$ and $a \leq b$, but $b \notin F_{a}$, So $F_{a}$ is not a filter. Also, we have $F_{b}=\{b, 1\}$ that is a filter, so the converse of Lemma 3.20 does not hold.

Proposition 3.22. $F_{1}=L \backslash\{0\}$.

Proof. It is clear that $F_{1} \subseteq L \backslash\{0\}$. Let $x \in L, x \neq 0$. Since $x \leq 1$, by Definition 3.2, $1 \in x \vee 1$. So $x \in F_{1}$ and it means that $(L \backslash\{0\}) \subseteq F_{1}$. So $F_{1}=L \backslash\{0\}$.

Theorem 3.23. Let $L$ be a dual distributive hyperlattice and $I$ be a nonempty subset of $L$. Define $F_{I}=\{x \in L: x \neq 0,1 \in x \vee a, \forall a \in I\}$. Then 
(i) $F_{I}$ is a filter of $L$.

(ii) $F_{I}=\bigcap_{a \in I} F_{a}$.

(iii) $F_{a} \cap F_{b}=F_{a \wedge b}$.

(iv) If $A \subseteq B$, then $F_{B} \subseteq F_{A}$.

Proof. We prove (iii), only. Then $x \in F_{a} \cap F_{b}$, if and only if $1 \in x \vee a$ and $1 \in x \vee b$, if and only if $1 \in(x \vee a) \wedge(x \vee b)=x \vee(a \wedge b)$, if and only if $1 \in F_{a \wedge b}$. Therefore, $F_{a} \cap F_{b}=F_{a \wedge b}$.

$F_{I}$ is called $I$-filter generated by $I$.

Corollary 3.24. Let $L$ be a dual distributive hyperlattice. Then the following conditions hold:

(i) If $a, b \in L$ and $a \leqslant b$, then $F_{a \vee b} \subseteq F_{b}$.

(ii) $F_{a} \supseteq F_{a \wedge b}$ and $F_{b} \supseteq F_{a \wedge b}$.

(iii) $\left(\left\{F_{a}: a \in L\right\}, \bigcap\right)$ is $a \wedge-$ semilattice.

Proof. (i): Let $a \leq b$. By Definition 3.2, $b \in a \vee b$ and it implies that $\{b\} \subseteq a \vee b$. By Theorem 3.23,(iv) we have: $F_{a \vee b} \subseteq F_{b}$.

(ii) and (iii) are obvious.

Example 3.25. (i) Let $L$ be the hyperlattice in Example 3.8 and $I=$ $\{a, 1\}$. We have $F_{a}=\{1\}$ and $F_{1}=\{a, 1\}$, then $F_{I}=F_{a} \cap F_{1}=$ $\{1\} \cap\{a, 1\}=\{1\}$.

(ii) Let $L$ be the hyperlattice in Example 3.21. We have $a \leq b$, but $F_{a} \nsubseteq F_{b}$ and $F_{b} \nsubseteq F_{a}$, since $F_{a}=\{a, 1\}$, and $F_{b}=\{b, 1\}$.

\section{Acknowledgements.}

The first author partially has been supported by the "Algebraic Hyperstructure Excellence, Tarbiat Modares University, Tehran, Iran" and "Research Center in Algebraic Hyperstructures and Fuzzy Mathematics, University of Mazandaran, Babolsar, Iran". The last author was supported within the project "VYZZKUMFVT (DZRO K-217)" supported by the Ministry of Defence, Czech Republic. 


\section{References}

[1] R. AMERI and S. HOSKOVA-MAYEROVA, Fuzzy Continuous Polygroups, APLIMAT 2016 - 15th Conference on Applied Mathematics, Proceedings. Slovak University of Technology in Bratislava, (2016), 13-19.

[2] R. AMERI, T. NOZARI, A Connection Between Categories of Multialgebras and Algebra, Ital. J. Pure Appl. Math., 27(2010), 201-208.

[3] R. AMERI, I. G. ROSENBERG, Congruences of Multialgebras, J. Mult.Valued Logic Soft Comput., 10(2009), 1-12.

[4] G. BIRKHOFF, Lattice Theory, American Mathematical Society Colloguium Publications $[M]$, Providence, Rhode Island, 1940.

[5] J. CHVAlinA, S. HOSKOVA-MAYEROVA, A. DEGHAN NEZHAD, General actions of hypergroups and some applications, An. Stiint. Univ. "Ovidius" Constanta Ser. Mat., 21(1), (2013), 59-82.

[6] J. CHVALINA, S. HOSKOVA-MAYEROVA, On certain proximities and preorderings on the transposition hypergroups of linear first-order partial differential operators, An. Stiint. Univ. "Ovidius" Constanta Ser. Mat., 22(1), (2014), 85-103.

[7] P. CORSINI, V. LEOREANU, Applications of Hyperstructure Theory, Kluwer Academic Publishers, Dordrecht, Hardbound, 2003.

[8] I. CRISTEA, S. JANCIC-RASOVIC, Compositions Hyperrings, An. Stiint. Univ. "Ovidius" Constanta Ser. Mat., 21(2), (2013), 81-94.

[9] I. CRISTEA, Regularity of Intuitionistic Fuzzy Relations on Hypergroupoids, An. Stiint. Univ. "Ovidius" Constanta Ser. Mat., 22(1), (2014), 105-119.

[10] I. CRISTEA, S. HOSKOVA, Fuzzy pseudotopological hypergroupoids, Iran. J. Fuzzy Syst., 6(4), (2009), 11-19.

[11] A. HOFMANN, S. HOSKOVA-MAYEROVA, V. TALHOFER, Usage of fuzzy spatial theory for modelling of terrain passability, Adv. in Fuzzy Syst. (2013), doi:10.1155/2013/506406.

[12] S. HOSKOVA, Topological hypergroupoids, Comput. Math. Appl., 64(9)(2012), 2845-2849.

[13] B. B. N. KOGUEP, C. NKUIMI, C. LELE, On fuzzy ideals of hyperlattice, Internat. J. Algebra, 2(2008), 739-750. 
[14] M. KONSTANTINIDOU, J. MITTAS, An introduction to the theory of hyperlattice, Math. Balcanica, 7(1977), 187-193.

[15] M. KRASNER, A class of hyperrings and hyperfields, Int. J. Math. and Math. Sci., 2(1983), 307-312.

[16] F. MARTY, Surene generalization de la notion de group, Eighth Congress Scandinaves, Stockholm, (1934), 45-49.

[17] M. NOVÁK, Some basic properties of EL-hyperstructures, European J. of Combin. 34(2013), 446-459.

[18] M. NOVÁK, $n$-ary hyperstructures constructed from binary quasi-orderer semigroups, An. Stiint. Univ. "Ovidius" Constanta Ser. Mat., 22(3), (2014), 147-168.

[19] A. RAHNEMAI-BARGHI, The prime ideal theorem for distributive hyperlattices, Ital. J. Pure Appl. Math., 10(2010), 75-78.

[20] S. RASOULI, B. DAVVAZ, Lattice derived from hyperlattices, Comm. in Algebra, 38(2010), 2720-2737.

[21] S. RASOULI, B. DAVVAZ, Construction and spectral topology on hyperlattice, Mediterr. J. Math., 7(2010), 249-262.

[22] D. SCHWEIGRT, Congruence Relation of multialgebra, Discrete Math., 53(1985), 249-253.

[23] V. TALHOFER, A. HOFMANN, S. HOSKOVA-MAYEROVA, Application of Fuzzy Membership Function in Mathematical Models for Estimation of Vehicle Trafficability in Terrain, APLIMAT 2015 - 14th Conference on Applied Mathematics, Proceedings, Slovak University of Technology in Bratislava, (2015), 711-719.

[24] T. VOUGIOUKLIS, Hyperstructures and their Representations, Hadronic Press, Inc., 1994.

Reza Ameri,

Department of Mathematics, Tehran University,

Tehran, Iran.

Email: rameri@ut.ac.ir

M. Amiri-Bideshki,

Department of Mathematics,

Payame-Noor University, Tehran, Tehran, Iran.

Email: amirimohsen61@yahoo.com 
Arsham Borumand Saeid,

Department of Pure Mathematics,

Faculty of Mathematics and Computer, Shahid Bahonar University of Ker-

man,

Kerman, Iran.

Email: arsham@uk.ac.ir

Sarka Hoskova-Mayerova,

Department of Mathematics and Physics,

University of Defence,

Kounicova 65, Brno, Czech Republic.

Email: sarka.mayerova@unob.cz 